\title{
nArgBP2-SAPAP-SHANK, the core postsynaptic triad associated with psychiatric disorders
}

\author{
Sang-Eun Lee', Jung Ah $\mathrm{Kim}^{2}$ and Sunghoe Chang ${ }^{1,2,3}$
}

\begin{abstract}
Despite the complex genetic architecture, a broad spectrum of psychiatric disorders can still be caused by mutation(s) in the same gene. These disorders are interrelated with overlapping causative mechanisms including variations in the interaction among the risk-associated proteins that may give rise to the specific spectrum of each disorder. Additionally, multiple lines of evidence implicate an imbalance between excitatory and inhibitory neuronal activity (E/I imbalance) as the shared key etiology. Thus, understanding the molecular mechanisms underlying E/l imbalance provides essential insight into the etiology of these disorders. One important class of candidate risk genes is the postsynaptic scaffolding proteins, such as nArgBP2, SAPAP, and SHANK that regulate the actin cytoskeleton in dendritic spines of excitatory synapses. This review will cover and discuss recent studies that examined how these proteins, especially nArgBP2, are associated with psychiatric disorders. Next, we propose a possibility that variations in the interaction among these proteins in a specific brain region might contribute to the onset of diverse phenotypes of psychiatric disorders.
\end{abstract}

\section{Introduction}

In neural circuits, the balance between excitatory and inhibitory neuronal activity (E/I balance) refers to the relative degree of excitatory and inhibitory neuronal activity $^{1}$. The amount of excitatory vs. inhibitory inputs modulates the excitability and function of a single neuron, and the integration of E/I drive in a group of neurons determines the strength of neuronal activity in a certain brain circuit of a particular brain region ${ }^{2,3}$. Thus, the ratio between excitation and inhibition has been recognized as a crucial measure for assessing brain fitness as its imbalance is implicated in many psychiatric disorders, including autism spectrum disorder (ASD) ${ }^{4}$, bipolar disorder $(\mathrm{BD})^{5}$, and schizophrenia $(\mathrm{SCZ})^{5,6}$. However, measuring the $\mathrm{E} / \mathrm{I}$ ratio is complicated because mixtures of disparate excitatory and inhibitory signals may represent various circuits in different brain regions. Even within a single

\footnotetext{
Correspondence: Sunghoe Chang (sunghoe@snu.ac.kr)

${ }^{1}$ Department of Physiology and Biomedical Sciences, Seoul National University College of Medicine, Seoul 03080, South Korea

${ }^{2}$ Interdisciplinary Program in Neuroscience, College of Natural Sciences, Seoul National University, Seoul 08826, South Korea

Full list of author information is available at the end of the article
}

circuit, a large flexibility between excitation and inhibition affects varying aspects of neuronal function. Despite these challenges, mutations in many of the postsynaptic proteins that affect the formation or maintenance of either excitatory or inhibitory synapses have been identified as a causative factor for the dysregulation of the $\mathrm{E} / \mathrm{I}$ balance in psychiatric disorders ${ }^{7}$. Indeed, recent studies indicate that assembly of the scaffolding proteins at excitatory synapses that regulate the actin cytoskeleton in dendritic spines is critical for maintaining the synaptic E/I balance and that either up- or downregulation of these molecules is implicated in various psychiatric disorders (Table 1).

In this review, we summarize recent studies that investigated how perturbations in the scaffolding proteins at excitatory synapses, especially focusing on nArgBP2, lead to an E/I imbalance. In addition, we propose a hypothesis that a shift in the balance of synaptic actin dynamics caused by altered interactions among nArgBP2SAPAP-SHANK, core-postsynaptic proteins, is an important factor in the manifestation of an $\mathrm{E} / \mathrm{I}$ imbalance in a specific brain region that accounts for various psychiatric disorders. 
Table 1 Table showing the SAPAP, SHANK, and nArgBP2 subtypes expressed in the brain and the corresponding CNS diseases

\begin{tabular}{|c|c|c|c|}
\hline Subtype & CNS disease & $\begin{array}{l}\text { Expression } \\
\text { level }\end{array}$ & References \\
\hline \multirow[t]{3}{*}{ SAPAP1 } & Schizophrenia & $\uparrow$ & 28 \\
\hline & Alzheimer's disease & $\downarrow$ & 88 \\
\hline & Major depressive disorder & $\downarrow$ & 29 \\
\hline \multirow[t]{4}{*}{ SAPAP2 } & Schizophrenia & $\uparrow$ & 32 \\
\hline & $\begin{array}{l}\text { Fragile } X \text { mental } \\
\text { retardation }\end{array}$ & $\uparrow$ & 89 \\
\hline & $\begin{array}{l}\text { Post-traumatic stress } \\
\text { disorder }\end{array}$ & $\downarrow$ & 30 \\
\hline & Autism spectrum disorder & $\downarrow$ & 31 \\
\hline \multirow[t]{4}{*}{ SAPAP3 } & Trichotillomania & $\downarrow$ & $35-37$ \\
\hline & $\begin{array}{l}\text { Obsessive compulsive } \\
\text { disorder }\end{array}$ & $\downarrow$ & $33,35-37$ \\
\hline & Parkinson's disease & $\downarrow$ & 90 \\
\hline & Schizophrenia & $\downarrow$ & 84,91 \\
\hline \multirow[t]{2}{*}{ SAPAP4 } & Cerebellar ataxia & $\downarrow$ & 92 \\
\hline & Bipolar disorder & $\downarrow$ & 93 \\
\hline \multirow[t]{3}{*}{ SHANK1 } & Autism spectrum disorder & $\downarrow$ & 50,51 \\
\hline & Schizophrenia & $\downarrow$ & 52,53 \\
\hline & Alzheimer's disease & $\downarrow$ & 94,95 \\
\hline \multirow[t]{5}{*}{ SHANK2 } & Autism spectrum disorder & $\downarrow$ & $31,55,96$ \\
\hline & Schizophrenia & $\downarrow$ & 57,97 \\
\hline & Bipolar disorder & $\downarrow$ & 97,98 \\
\hline & Intellectual disability & $\downarrow$ & $31,54-56,96$ \\
\hline & Alzheimer's disease & $\uparrow$ & 99 \\
\hline \multirow[t]{8}{*}{ SHANK3 } & Autism spectrum disorder & $\downarrow$ & $86,100-104$ \\
\hline & Epilepsy & $\downarrow$ & 105,106 \\
\hline & $\begin{array}{l}\text { Obsessive compulsive } \\
\text { disorder }\end{array}$ & $\downarrow$ & 107,108 \\
\hline & $\begin{array}{l}\text { Attention-deficit/ } \\
\text { hyperactivity disorder }\end{array}$ & $\uparrow$ & 104,109 \\
\hline & $\begin{array}{l}\text { Phelan-McDermid } \\
\text { syndrome }\end{array}$ & $\downarrow$ & $46,47,110-112$ \\
\hline & Schizophrenia & $\downarrow$ & $\begin{array}{l}58,60,87,108,113- \\
115\end{array}$ \\
\hline & Bipolar disorder & $\uparrow$ & $59,111,116,117$ \\
\hline & Alzheimer's disease & $\downarrow$ & 94,95 \\
\hline \multirow[t]{2}{*}{ nArgBP2 } & Bipolar disorder & $\downarrow$ & $73,74,118$ \\
\hline & Intellectual disability & $\downarrow$ & 65 \\
\hline
\end{tabular}

The upward or downward arrow signifies up- or downregulation/mutation, respectively

\section{Dendritic spine regulation as a common mechanism of psychiatric disorders}

Most studies on excitatory synaptic transmission linked to $\mathrm{E} / \mathrm{I}$ imbalance are focused on the proteins that regulate dendritic spines. Dendritic spines are tiny protrusions on the dendrites and serve as a structural unit, where excitatory synaptic transmission occurs ${ }^{8}$. Spines integrate excitatory signals into the output passed on to another neuron in the brain circuit $^{9}$. Spines are classified according to their morphological features such as shape and size since their morphology is highly correlated with their functional properties. Spines actively participate in the formation of synapses and neuronal circuits during development ${ }^{10}$, whereas activity-dependent spine maintenance or elimination is important for the remodeling of established neuronal circuits during postnatal and adolescent periods ${ }^{11,12}$. Persistent changes in synaptic strength during synaptic plasticity also involve structural changes of spines. Indeed, spines are morphologically regulated to meet the dynamic demands of the brain and are intimately linked to higher brain function ${ }^{10}$. Molecular pathways involving the regulation of spines include cytoskeletal remodeling, trans-synaptic adhesion, receptor trafficking, protein translation, ubiquitination, and gene expression $^{13,14}$.

The cytoplasm of dendritic spines is enriched with highly branched filamentous actin (F-actin) located exactly beneath the postsynaptic density (PSD). The actin cytoskeleton stabilizes and modulates the dynamic dendritic spine structure in response to neuronal activity ${ }^{15,16}$, and the regulation of such dynamics is critical for the morphological changes, maturation, and physiological function of spines ${ }^{17}$. A recent study using superresolution imaging showed that the actin cytoskeleton is unevenly distributed and rapidly reorganized within synapses $^{18}$.

The dendritic spine contains a large number of scaffolding proteins, including SHANK (or Pro-SAP), SAPAP (or GKAP), and nArgBP2, which share highly conserved domains for protein-protein interactions. These proteins can interact with multiple proteins simultaneously to physically connect PSD components and organize binding partners into a functional unit to enhance postsynaptic signaling ${ }^{19}$ (Fig. 1). The specialized functional network composed of these proteins provides a direct link between membrane receptors, cell adhesion molecules, and the actin cytoskeleton, thus facilitating activity-dependent dendritic remodeling, which is critical for synaptogenesis and synaptic plasticity ${ }^{15,16}$.

Anatomical analysis often reveals altered dendritic spine morphology in the brains of patients with SCZ, intellectual disabilities, and $\mathrm{ASDs}^{20}$. Indeed, most genetic human risk factors for these disorders are associated with the signaling of actin cytoskeleton dynamics in dendritic 


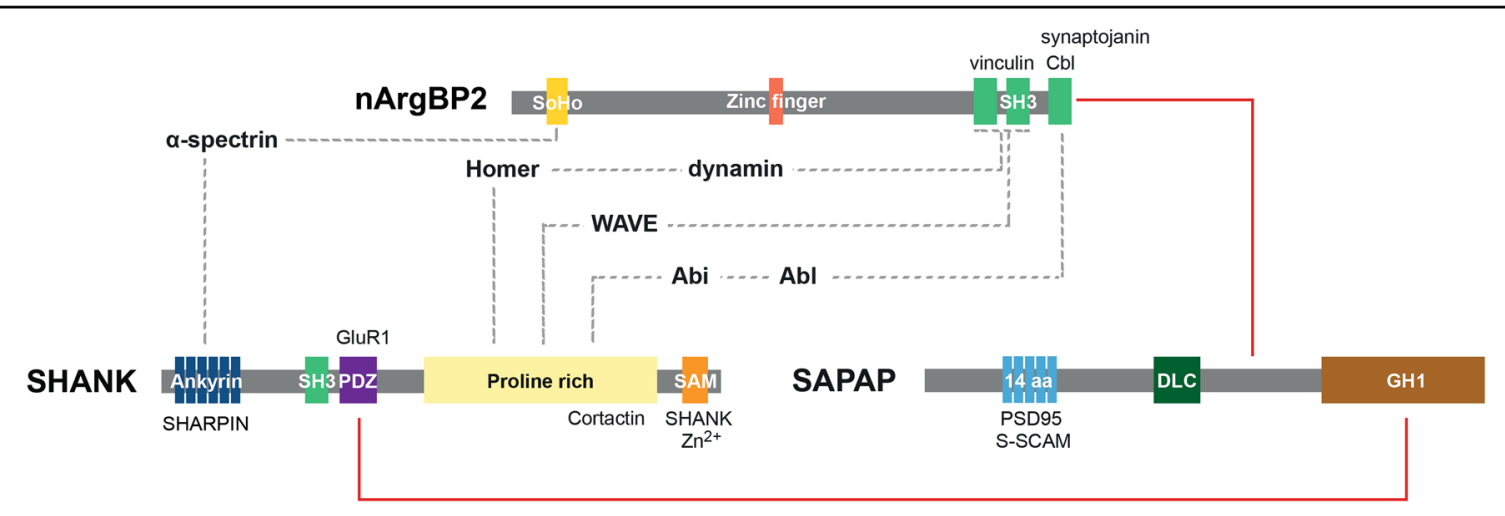

Fig. 1 The domain architectures of nArgBP2, SHANK, and SAPAP. Proteins listed with each of the domains indicate the known binding partners. Only a subset of known interacting proteins is shown. nArgBP2, SHANK, and SAPAP interact with each other either directly via specific domains (red lines) or indirectly through common binding partners (broken lines). SAPAP interacts with nArgBP2 via its proline-rich region between the DLC and GH1 regions. 14 aa 14 amino acid domain, Abi Abelson interacting protein-1, DLC dynein light chain, GH1 GKAP homology 1, nArgBP2 neural Arg binding protein 2, PDZ PSD95 Dlg1 and zo-, 1 PSD95 postsynaptic density protein 95, SAM sterile alpha motif, SAPAP SAP90/PSD95-associated protein, SHANK SH3 and multiple ankyrin repeat domains, SH3 Src homology 3 domain, SoHo sorbin homology domain, S-SCAM synaptic scaffolding molecule, WAVE WASP (Wiskott-Aldrich syndrome protein)-family verprolin homologous protein

spines ${ }^{21}$. Accordingly, various studies have reported that numerous psychiatric disorders are characterized by dendritic abnormalities, including aberrant spine density and morphology, which are most likely to significantly affect excitatory drive and subsequently $\mathrm{E} / \mathrm{I}$ balance in the brain circuit ${ }^{22}$.

\section{Core postsynaptic proteins associated with psychiatric disorders \\ SAPAP}

The four members of the guanylate kinase-associated protein (GKAP) family were originally identified as proteins that interact with the GK domain of PSD $95^{23}$ and named SAP90/PSD95-associated protein (SAPAP) $1-4^{24}$. SAPAPs consist of a 14-amino acid repeat domain, a dynein light chain (DLC) domain, and a GKAP homology (GH1) domain. As a scaffold protein, SAPAP binds to PSD95, synaptic scaffolding molecule (S-SCAM), nArgBP2 and dynein light chain via its $\mathrm{N}$-terminal domain $^{24-26}$, while it interacts with the PDZ domain of ProSAPs/SHANKs through its C-terminal domain ${ }^{27}$ (Fig. 1).

\section{Implications of SAPAPs in psychiatric disorders}

A previous study found that SAPAP1 immunoreactivity is significantly increased in the nucleus accumbens of unmedicated patients with $\mathrm{SCZ}^{28}$. SAPAP1 is also implicated in recurrent major depressive disorder according to a report from a study of Mexican American individuals ${ }^{29}$. Screening of genomic DNA methylation patterns in a rat post-traumatic stress disorder (PTSD) model revealed that an increase in a specific methylation site causes a reduction in SAPAP2 gene expression, thus pinpointing SAPAP2 as a possible target for PTSD ${ }^{30}$. Some common and rare genetic variants of SAPAP2 have also been found to be associated with $\mathrm{ASD}^{31}$, and increased SAPAP2 gene expression contributes to the pathogenesis of $\mathrm{SCZ}^{32}$. Genetic deletion of SAPAP3 in mice causes behavioral abnormalities including increased anxiety and compulsive self-grooming ${ }^{33}$, which are similar to symptoms exhibited by human patients with obsessive compulsive disorder (OCD) and phenotypes exhibited by other genetic animal models of OCD-like behaviors ${ }^{19,34}$. OCD-like behaviors in SAPAP3-null mice have been indicated in several recent human genetics studies of the role of SAPAP3 in obsessive compulsive spectrum disorder (OCSD) $)^{35-37}$.

\section{SHANK}

The SH3 and multiple ankyrin repeat domains (SHANK) family is a group of scaffold proteins that contain ankyrin repeat domains, a SH3 domain, one PDZ domain, a proline-rich region (PRD), and a sterile alpha motif (SAM) domain ${ }^{38}$. The ankyrin repeat domain, containing six ankyrin repeats, interacts with the PSD protein SHARPIN $^{39}$ and binds to the cytoskeleton through an interaction with $\alpha$-spectrin (also known as $\alpha$-fodrin $)^{40}$. The PDZ domain of SHANK interacts with SAPAP $1^{41}$ and the GluR1 subunit of AMPA receptors ${ }^{42}$ and, therefore, is important for dendritic spine formation and synaptic transmission. The PRD binds to Homer ${ }^{43}$ and $\operatorname{cortactin}^{41}$, and such an interaction is important for cytoskeleton regulation as well as synaptic transmission and plasticity. The C-terminal SAM domain is important for self-multimerization of the protein ${ }^{41}$ and is required for the localization of SHANK to the PSD $^{44}$ (Fig. 1). SHANK binds to SAPAP, which in turn binds to PSD95 to form a PSD95/SAPAP/SHANK postsynaptic complex ${ }^{45}$. 


\section{Implications of SHANKs in psychiatric disorders}

SHANK genes were first identified in studies of Phelan-McDermid syndrome (PMS), a neurodevelopmental disorder that is caused by chromosome 22q13.3 deletion and characterized by autistic-like behaviors, hypotonia, and delayed or absent speech ${ }^{46-48}$. Moreover, mutations or disruptions in the SHANK gene family account for $\sim 1 \%$ of all patients with $\mathrm{ASD}^{49}$.

SHANK1 (also known as ProSAP3) deletions and mutations have been reported to cause broad autism phenotypes in males ${ }^{50}$. A recent study also found a de novo SHANK1 mutation in a female with ASD $^{51}$. Lennerts et al. found that the T allele of SNP rs3810280 in the SHANK1 promoter is associated with reduced auditory working memory in patients with $\mathrm{SCZ}^{52}$, and Fromer et al. reported a de novo SHANK1 frameshift mutation in a female schizophrenia patient ${ }^{53}$.

Several studies have identified deleterious mutations of SHANK2 (also known as ProSAP1) in patients with ASD and mild/moderate intellectual disability (ID) $)^{31,54-56}$. Missense variants of SHANK2 identified in SCZ patients correspond to a mutation in hippocampal neurons that leads to a loss of presynaptic contacts and reduced clustering of SHANK2 at synapses ${ }^{57}$.

SHANK3 (also known as ProSAP2) is the best studied among the three SHANK family members, and its mutant mice exhibit autistic-like behaviors, including impaired social interaction and repetitive behaviors. SHANK3 was first recognized in $\mathrm{PMS}^{48}$ and later in $\mathrm{ASD}^{46}$ with symptoms that include ID, autistic behaviors, hypotonia, and significant language impairment ${ }^{58}$. In addition, duplication of the SHANK3 gene is often found in patients diagnosed with $\mathrm{BD}$, and mice with an extra copy of SHANK3 exhibit manic-like phenotypes ${ }^{59}$. A recent study further found that mice with the ASD-linked InsG3680 mutation in SHANK3 show defective synaptic transmission in the striatum and impaired juvenile social interaction, coinciding with the early onset of ASD symptoms, while adult mice carrying the schizophrenia-linked R1117X mutation show profound synaptic defects in the prefrontal cortex and social dominance behavior ${ }^{60}$.

\section{nArgBP2}

\section{Domain structure of $n A r g B P 2$}

nArgBP2 is a neural variant of ArgBP2 (Arg binding protein 2, also known as SORBS2) and was first cloned from the rat brain as an SAPAP-binding protein ${ }^{26}$. The full-length protein is composed of 1196 amino acids containing multiple domains for protein-protein interaction including a sorbin homology ( $\mathrm{SoHo}$ ) domain in the $\mathrm{N}$-terminal region and three $\mathrm{Src}$ homology 3 (SH3) domains in the C-terminal region ${ }^{61}$. nArgBP2 has one zinc-finger motif in the middle region (Fig. 1).

\section{Expression patterns of nArgBP2 mRNAs and proteins}

The SORBS2 gene encodes multiple transcripts including four ArgBP2 isoforms ( $\alpha, \beta, \gamma$, and $\delta$ isoform) and nArgBP2 through alternative RNA splicing ${ }^{26,62-64}$. ArgBP2/nArgBP2 isoforms are widely expressed in human tissues and are especially abundant in the brain, heart, pancreas, and colon ${ }^{63}$. A transcript corresponding to an nArgBP2-specific exon is detected only in the brain tissue and exclusively in neurons ${ }^{26,65}$. In the brain, nArgBP2 mRNA is highly expressed in the isocortex, hippocampus, cortical subplate, striatum, thalamus, and hypothalamus, the majority of which are regions of the brain associated with $\mathrm{BD}^{66,67}$. At the protein level, nArgBP2 is only detected in the brain with a high expression level in the cortex, amygdala, and dentate gyrus (DG) and moderate expression level in the striatum, lateral habenula, and thalamus. Within cortical regions, immunoreactivity for $\mathrm{nArgBP} 2$ is more intense in layers I-III in the neocortex and layer I of the piriform cortex. In the DG, $\mathrm{nArgBP} 2$ is specifically enriched at the edge of the molecular layer ${ }^{26,65}$.

\section{Protein interactions of $n A r g B P 2$}

nArgBP2 is a scaffold protein that controls the balance between adhesion and motility by modulating the function of multiple signaling pathways that converge on the actin cytoskeleton ${ }^{61}$. The N-terminal SoHo domain interacts with $\alpha 2$-spectrin, and the three $\mathrm{SH} 3$ domains have partially overlapping binding partners, with the second $\mathrm{SH} 3$ having the most interactors including $\mathrm{SAPAP}^{26}$, synaptojanin $1 / 2^{68}$, vinculin ${ }^{26}, \mathrm{Abl}^{63}, \mathrm{Cbl}^{69}$, and dynamin $1 / 2^{61}$. Other interactors include the actinregulating Wiskott-Aldrich syndrome protein family verprolin homologous protein (WAVE) 1 and 2 and components of a WAVE regulatory complex (PIR121 and Nap1 $)^{61,70,71}$.

ArgBP2 mediates the formation of a complex containing $\mathrm{Abl}$ and $\mathrm{Cbl}$, which promotes the Abl-dependent phosphorylation of $\mathrm{Cbl}$ and the ubiquitination by $\mathrm{Cbl}$ of both $\mathrm{Abl}$ and $\mathrm{ArgBP}^{69}$. In the brain, Abl interacts with Abelson interacting protein-1 (Abi-1), which binds to SHANK and is essential for dendrite morphogenesis and synapse formation ${ }^{72}$. nArgBP2 interacts with SHANK either directly or indirectly through SAPAP ${ }^{26}$, suggesting a possible network formation among nArgBP2, SAPAP, and SHANK.

\section{Synaptic localization of $n A r g B P 2$}

In rat brain subcellular fractions, $\mathrm{nArgBP} 2$ is detected in the cytosol of the synaptosome but is also enriched in the PSD fraction ${ }^{26}$. Immunocytochemistry analysis with specific nArgBP2 antibodies indicates that nArgBP2 


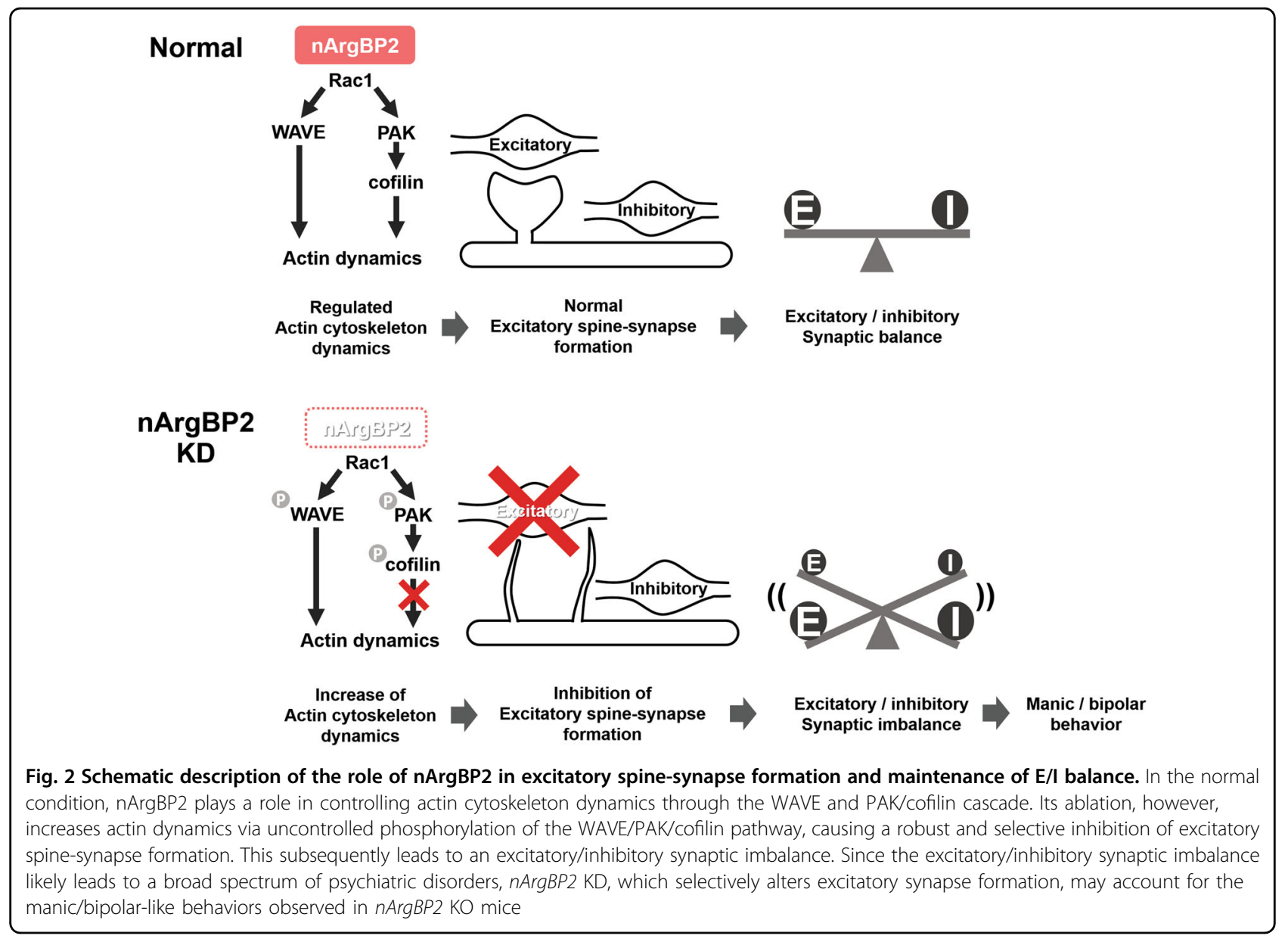

colocalizes with F-actin and is enriched in both dendritic and axonal terminals in early developing neurons ${ }^{65}$. In mature neurons, $\mathrm{nArgBP} 2$ is mainly enriched in dendrites, especially in dendritic spines in which it colocalizes with the excitatory postsynaptic scaffolding protein PSD95 $5^{65,73}$. Dendritic clusters of nArgBP2 are also juxtaposed with vesicular glutamate transporter 1 (vGLUT1) puncta in spiny neurons ${ }^{73}$. In contrast, nArgBP2 rarely overlaps with the inhibitory postsynaptic scaffolding protein gephyrin in spiny neurons and is almost absent in aspiny gephyrin-positive neurons ${ }^{73}$. Collectively, these observations show that $\mathrm{nArgBP} 2$ primarily localizes to excitatory synapses in spiny pyramidal neurons and plays a specific functional role in these excitatory synapses.

\section{nArgBP2 in dendritic spine formation}

Recently, Zhang et al. found that dendritic arborization of DG granule cells in $n A r g B P 2 K O$ mice shows much less complexity with a significantly reduced number of dendritic branch points than those in wild-type (WT) littermates $^{65}$. We also found that the density of total dendritic protrusions and the proportion of mushroom-shaped spines are dramatically decreased with $n A r g B P 2$ knockdown $(\mathrm{KD})^{73}$. Additionally, the expression level of the active form of WAVE1 is increased in $n A r g B P 2 \mathrm{KD}^{73}$, and Rac1 activity is enhanced, leading to p21-activated kinase (PAK) activation and cofilin inactivation. Dendritic spines in KD neurons are highly motile, and the pool of dynamic actin downstream of Rac1 activation is significantly increased accordingly ${ }^{73}$.

\section{$n A r g B P 2$ in the regulation of excitatory synaptic formation and transmission}

$n A r g B P 2 \mathrm{KD}$ causes a specific and dramatic reduction in the number of spine-synapses, which is consistent with the decreased number of mushroom-shaped spines in $\mathrm{KD}$ neurons $^{73}$ (Fig. 2). Furthermore, the mean frequency of miniature excitatory postsynaptic currents (mEPSCs) is significantly decreased compared to that in control neurons, while that of miniature inhibitory postsynaptic currents (mIPSCs) is not affected ${ }^{73}$. Consistently, in $n A r g B P 2 \mathrm{KO}$ mice, the mean frequency of mEPSCs is also significantly reduced compared with that in WT littermates ${ }^{65}$. These findings indicate that nArgBP2 functions to regulate spine morphogenesis and subsequent spinesynapse formation at glutamatergic synapses and that its 
ablation causes a robust and selective inhibition of excitatory synapse formation by controlling actin cytoskeleton dynamics (Fig. 2).

\section{Behavioral phenotypes of nArgBP2 KO mice and implications of $n A r g B P 2$ in psychiatric disorders}

$n A \operatorname{ArBP} 2 \mathrm{KO}$ mice have been reported to display manic/bipolar-like behavior including increased activity, compulsive/repetitive behavior, risk-taking behavior, hedonistic behavior, and anti-depressant-like behavior, resembling many aspects of symptoms in BD patients $^{74}$. Additional behavioral phenotypes include altered long-term but not short-term object recognition memory, as well as impaired contextual fear memory $^{65}$. A specific defect in contextual fear memory but not toned fear memory implies that nArgBP2 may have a more important role in the hippocampus than in the amygdala ${ }^{75}$. $n \operatorname{ArgBP} 2 \mathrm{KO}$ mice also show a reduced dendritic complexity of DG granule cells ${ }^{65}$, which has also been shown in other mouse models of ID $^{76,77}$, supporting the link between the mutation of $n A r g B P 2$ and $\mathrm{ID}^{53} \cdot \mathrm{nArgBP} 2$ de novo mutations are also found in SCZ patients ${ }^{53}$.

\section{Postsynaptic triad of nArgBP2-SAPAP-SHANK, a convergent pathway of psychiatric disorders}

Data from a large-scale study revealed that there are a substantial number of individuals who simultaneously meet the criteria for two or more psychiatric disorders. For example, comorbid $\mathrm{BD}$ and $\mathrm{OCD}$ are relatively common among patients with a primary diagnosis of $\mathrm{BD}^{78}$. Studies have also found that as many as $27 \%$ of those with ASD also have symptoms of $\mathrm{BD}^{79,80}$. Similarly, there is a strong positive correlation between measures of the severity of both depression and anxiety symptoms ${ }^{81}$. Indeed, recent genome-wide genetic studies have further identified overlapping risk genes across the major psychiatric disorders, including SCZ, BD, major depressive disorder, and $\mathrm{ASD}^{80,82-84}$. These results indicate that dysregulation of the same gene can cause different types of disorders, further suggesting the existence of a convergent mechanism for various psychiatric disorders with multiple etiologies.

Recent studies suggest that deletion or overexpression of postsynaptic proteins, such as nArgBP2, SAPAP, and SHANK, leads to behavioral phenotypes that are similar to symptoms observed in human ASD, BD, SCZ, and $\mathrm{OCD}^{59,60,74,85}$. Deletion of SAPAP causes OCD-like behaviors $^{33}$, some genetic variants of SAPAP2 are associated with $\mathrm{ASD}^{31}$, and increased SAPAP2 expression contributes to the pathogenesis of $\mathrm{SCZ}^{32}$. In addition, SHANK3 deletion or mutation is associated with $\mathrm{ASD}^{86}$, and its duplication or other mutations result in BD-like manic behaviors ${ }^{59}$ or SCZ-like behaviors ${ }^{87}$. Deletion of
nArgBP2 causes ID and BD-like symptoms ${ }^{65,74}$. Interestingly, SHANK binds to the GH1 domain of SAPAP directly via its PDZ domain. SAPAP binds to the SH3 domain of $n A r g B P 2$ via its proline-rich region. nArgBP2 and SHANK share a number of proteins as common binding partners (Fig. 1). Therefore, these three major postsynaptic proteins indeed interact with each other in dendritic spines of excitatory synapses such that the high incidence of comorbidity among psychiatric disorders is comprehensible. Although the underlying molecular mechanisms that connect the aberrant expression of these genes and behavioral consequences are still far from elucidated, E/I imbalance caused by dysregulation of the actin cytoskeleton in dendritic spines has been suggested as a shared key molecular mechanism.

Here, we propose a hypothesis that nArgBP2, SAPAP, and SHANK form a core scaffolding triad and together regulate actin cytoskeleton in dendritic spines. Different levels of expression or activity of this core triad may result in different phenotypes of psychiatric disorders in a brain region-specific manner, thus asserting that actin regulation by the core triad in dendritic spines is a convergent mechanism for psychiatric disorders (Fig. 3). Similar to each point of an equilateral triangle, these three proteins interact with each other to form a structural and functional postsynaptic scaffold that organizes multiple protein interactions to regulate the actin cytoskeleton in dendritic spines. Altered expression or mutation of either one or more of the proteins in the core triad in a specific brain region would break the balance of the interactome and induce defects in actin regulation, leading to spine structure abnormalities and, subsequently, to defects in the excitatory synaptic integrity. Depending on which individual protein is affected and, accordingly, which distinct protein network is disrupted, the $\mathrm{E} / \mathrm{I}$ balance may be differently altered at molecular, synaptic, and circuit levels in a brain region-specific manner, and these differences will be the contributing factors for the diverse phenotypes of psychiatric disorders. Through the combination of genetic approaches and recently developed imaging techniques, the identification of critical brain regions and neuronal types in which the core triad protein complex of nArgBP2, SAPAP, and SHANK differentially regulates synaptic function will help us to test and advance this tempting hypothesis.

\section{Conclusion}

Considering the high diversity and complexity of psychiatric disorders, it is surprising that many risk genes are shared by multiple psychiatric disorders and that many of these risk genes are regulators of actin cytoskeleton signaling in dendritic spines. Consistently, accumulating evidence suggests that alterations in dendritic spine morphology are a shared molecular etiology across different 


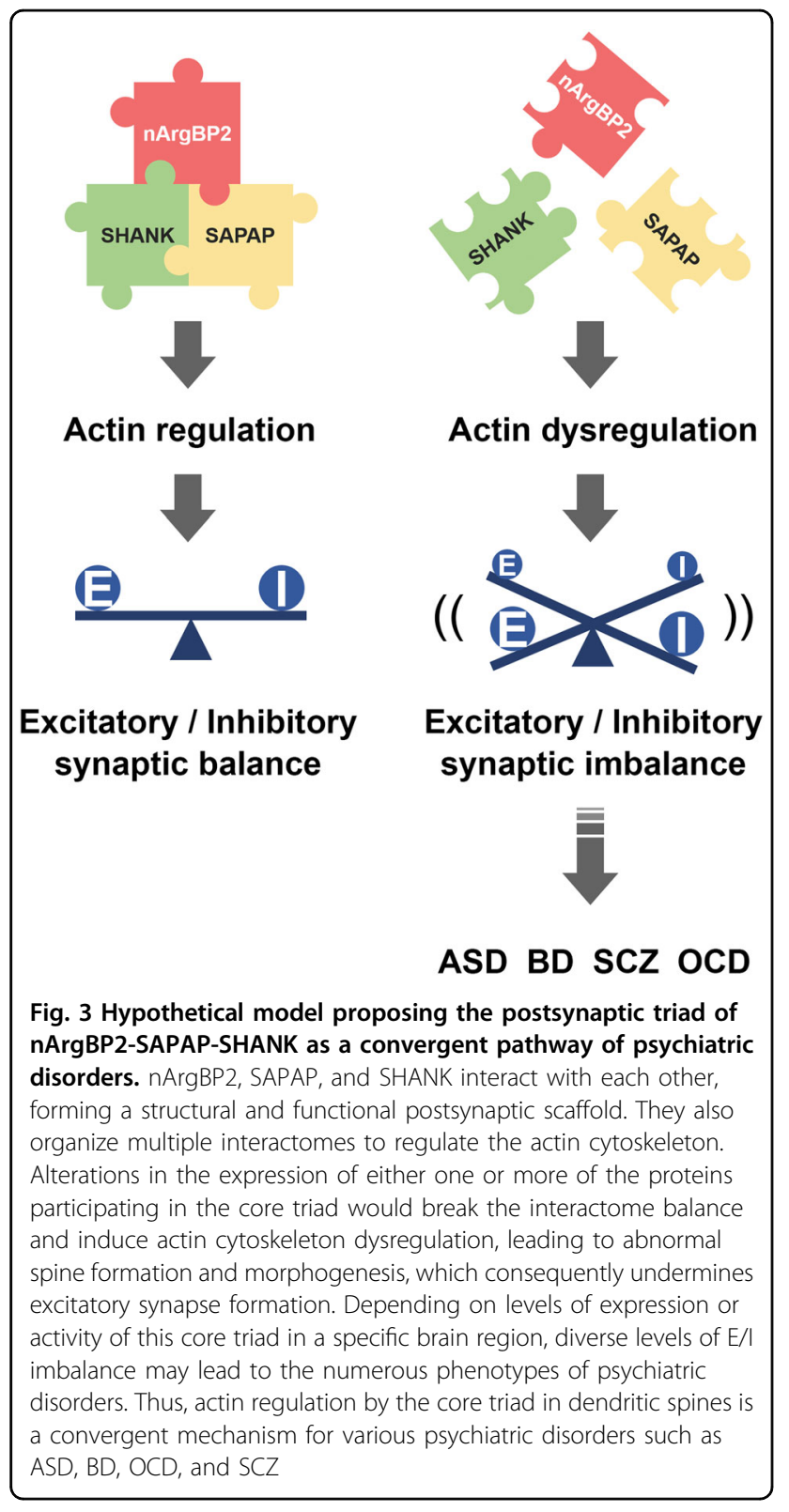

psychiatric disorders. We propose that nArgBP2, SAPAP, and SHANK form the core scaffolding triad to precisely mediate actin regulation in dendritic spines and that this serves as a convergent mechanism for different psychiatric disorders. Further studies on the brain region-specific regulation of actin dynamics by the core triad will be necessary to better understand the molecular mechanisms of various psychiatric disorders.

\section{Acknowledgements}

This research was supported by grants from the Brain Research Program (NRF2015M3C7A1028790 and 2017M3C7A1044957-8) to S.C. through the National Research Foundation of Korea funded by the Ministry of Science, ICT \& Future Planning, Republic of Korea. This work was also supported by the Education and Research Encouragement Fund of Seoul National University Hospital.

\section{Author details}

'Department of Physiology and Biomedical Sciences, Seoul National University College of Medicine, Seoul 03080, South Korea. ${ }^{2}$ Interdisciplinary Program in Neuroscience, College of Natural Sciences, Seoul National University, Seoul 08826, South Korea. ${ }^{3}$ Neuroscience Research Institute, Seoul National University College of Medicine, Seoul 03080, South Korea

\section{Authors' contributions}

S.-E.L. and S.C. contributed to the literature search. S.-E.L., J.A.K., and S.C. discussed all content of the manuscript and wrote the manuscript.

\section{Conflict of interest}

The authors declare that they have no conflict of interest.

\section{Publisher's note}

Springer Nature remains neutral with regard to jurisdictional claims in published maps and institutional affiliations.

Received: 14 November 2017 Accepted: 29 November 2017.

Published online: 9 April 2018

\section{References}

1. Penzes, P., Buonanno, A., Passafaro, M., Sala, C. \& Sweet, R. A. Developmental vulnerability of synapses and circuits associated with neuropsychiatric disorders. J. Neurochem. 126, 165-182 (2013).

2. Bourgeron, T. A synaptic trek to autism. Curr. Opin. Neurobiol. 19, 231-234 (2009).

3. Zoghbi, H. Y. Postnatal neurodevelopmental disorders: meeting at the synapse? Science $\mathbf{3 0 2}$, 826-830 (2003).

4. Rubenstein, J. L. \& Merzenich, M. M. Model of autism: increased ratio of excitation/inhibition in key neural systems. Genes Brain Behav. 2, 255-267 (2003).

5. Benes, F. M. \& Berretta, S. GABAergic interneurons: implications for understanding schizophrenia and bipolar disorder. Neuropsychopharmacology $\mathbf{2 5}$ $1-27$ (2001).

6. Hoftman, G. D., Datta, D. \& Lewis, D. A. Layer 3 excitatory and inhibitory circuitry in the prefrontal cortex: developmental trajectories and alterations in schizophrenia. Biol. Psychiatry 81, 862-873 (2017).

7. Lee, E., Lee, J. \& Kim, E. Excitation/inhibition imbalance in animal models of autism spectrum disorders. Biol. Psychiatry 81, 838-847 (2017).

8. Bourne, J. N. \& Harris, K. M. Balancing structure and function at hippocampal dendritic spines. Annu. Rev. Neurosci. 31, 47-67 (2008).

9. Spruston, N. Pyramidal neurons: dendritic structure and synaptic integration. Nat. Rev. Neurosci. 9, 206-221 (2008).

10. Holtmaat, A. \& Svoboda, K. Experience-dependent structural synaptic plasticity in the mammalian brain. Nat. Rev. Neurosci. 10, 647-658 (2009).

11. Alvarez, V. A. \& Sabatini, B. L. Anatomical and physiological plasticity of dendritic spines. Annu. Rev. Neurosci. 30, 79-97 (2007).

12. Zuo, Y., Lin, A., Chang, P. \& Gan, W. B. Development of long-term dendritic spine stability in diverse regions of cerebral cortex. Neuron 46, 181-189 (2005).

13. Penzes, P. \& Jones, K. A. Dendritic spine dynamics--a key role for kalirin-7. Trends Neurosci. 31, 419-427 (2008).

14. Tada, T. \& Sheng, M. Molecular mechanisms of dendritic spine morphogenesis. Curr. Opin. Neurobiol. 16, 95-101 (2006).

15. Bosch, M. et al. Structural and molecular remodeling of dendritic spine substructures during long-term potentiation. Neuron 82, 444-459 (2014).

16. Frost, N. A., Kerr, J. M., Lu, H. E. \& Blanpied, T. A. A network of networks: cytoskeletal control of compartmentalized function within dendritic spines. Curr. Opin. Neurobiol. 20, 578-587 (2010).

17. Korobova, F. \& Svitkina, T. Molecular architecture of synaptic actin cytoskeleton in hippocampal neurons reveals a mechanism of dendritic spine morphogenesis. Mol. Biol. Cell 21, 165-176 (2010).

18. Urban, N. T., Willig, K. I., Hell, S. W. \& Nagerl, U. V. STED nanoscopy of actin dynamics in synapses deep inside living brain slices. Biophys. J. 101 1277-1284 (2011). 
19. Ting, J. T., Peca, J. \& Feng, G. Functional consequences of mutations in postsynaptic scaffolding proteins and relevance to psychiatric disorders. Annu. Rev. Neurosci. 35, 49-71 (2012).

20. Zoghbi, H. Y. \& Bear, M. F. Synaptic dysfunction in neurodevelopmental disorders associated with autism and intellectual disabilities. Cold Spring Harb. Perspect. Biol. 4, a009886 (2012).

21. Sala, C. \& Segal, M. Dendritic spines: the locus of structural and functional plasticity. Physiol. Rev. 94, 141-188 (2014).

22. Penzes, P., Cahill, M. E., Jones, K. A., VanLeeuwen, J. E. \& Woolfrey, K. M. Dendritic spine pathology in neuropsychiatric disorders. Nat. Neurosci. 14 285-293 (2011).

23. Kim, E. et al. GKAP, a novel synaptic protein that interacts with the guanylate kinase-like domain of the PSD-95/SAP90 family of channel clustering molecules. J. Cell Biol. 136, 669-678 (1997).

24. Takeuchi, M. et al. SAPAPs. A family of PSD-95/SAP90-associated proteins localized at postsynaptic density. J. Biol. Chem. 272, 11943-11951 (1997).

25. Naisbitt, S. et al. Interaction of the postsynaptic density-95/guanylate kinase domain-associated protein complex with a light chain of myosin- $\mathrm{V}$ and dynein. J. Neurosci. 20, 4524-4534 (2000).

26. Kawabe, H. et al. nArgBP2, a novel neural member of ponsin/ArgBP2/vinexin family that interacts with synapse-associated protein 90/postsynaptic density-95-associated protein (SAPAP). J. Biol. Chem. 274, 30914-30918 (1999).

27. Verpelli, C., Schmeisser, M. J., Sala, C. \& Boeckers, T. M. Scaffold proteins at the postsynaptic density. Adv. Exp. Med. Biol. 970, 29-61 (2012).

28. Kajimoto, Y. et al. Synapse-associated protein 90/postsynaptic density-95associated protein (SAPAP) is expressed differentially in phencyclidinetreated rats and is increased in the nucleus accumbens of patients with schizophrenia. Neuropsychopharmacology 28, 1831-1839 (2003).

29. Mathias, S. R. et al. Recurrent major depression and right hippocampal volume: a bivariate linkage and association study. Hum. Brain Mapp. 37 191-202 (2016).

30. Chertkow-Deutsher, Y., Cohen, H., Klein, E. \& Ben-Shachar, D. DNA methylation in vulnerability to post-traumatic stress in rats: evidence for the role of the post-synaptic density protein Dlgap2. Int. J. Neuropsychopharmacol. 13, 347-359 (2010).

31. Pinto, D. et al. Functional impact of global rare copy number variation in autism spectrum disorders. Nature 466, 368-372 (2010).

32. Li, J. M. et al. Role of the DLGAP2 gene encoding the SAP90/PSD-95-associated protein 2 in schizophrenia. PLOS ONE 9, e85373 (2014).

33. Welch, J. M. et al. Cortico-striatal synaptic defects and OCD-like behaviours in Sapap3-mutant mice. Nature 448, 894-900 (2007).

34. Shmelkov, S. V. et al. Slitrk5 deficiency impairs corticostriatal circuitry and leads to obsessive-compulsive-like behaviors in mice. Nat. Med. 16, 598-602 (2010).

35. Bienvenu, O. J. et al. Sapap3 and pathological grooming in humans: results from the OCD collaborative genetics study. Am. J. Med. Genet. B Neuropsychiatr. Genet. 150B, 710-720 (2009).

36. Boardman, L. et al. Investigating SAPAP3 variants in the etiology of obsessivecompulsive disorder and trichotillomania in the South African white population. Compr. Psychiatry 52, 181-187 (2011).

37. Zuchner, S. et al. Multiple rare SAPAP3 missense variants in trichotillomania and OCD. Mol. Psychiatry 14, 6-9 (2009).

38. Lim, S. et al. Characterization of the Shank family of synaptic proteins. Multiple genes, alternative splicing, and differential expression in brain and development. J. Biol. Chem. 274, 29510-29518 (1999).

39. Lim, S. et al. Sharpin, a novel postsynaptic density protein that directly interacts with the shank family of proteins. Mol. Cell. Neurosci. 17, 385-397 (2001).

40. Bockers, T. M. et al. Synaptic scaffolding proteins in rat brain. Ankyrin repeats of the multidomain Shank protein family interact with the cytoskeletal protein alpha-fodrin. J. Biol. Chem. 276, 40104-40112 (2001).

41. Naisbitt, S. et al. Shank, a novel family of postsynaptic density proteins that binds to the NMDA receptor/PSD-95/GKAP complex and cortactin. Neuron 23, 569-582 (1999)

42. Uchino, S. et al. Direct interaction of post-synaptic density-95/Dlg/ZO-1 domain-containing synaptic molecule Shank3 with GluR1 alpha-amino-3hydroxy-5-methyl-4-isoxazole propionic acid receptor. J. Neurochem. 97, 1203-1214 (2006).

43. Tu, J. C. et al. Coupling of mGluR/Homer and PSD-95 complexes by the Shank family of postsynaptic density proteins. Neuron 23, 583-592 (1999).
44. Boeckers, T. M. et al. C-terminal synaptic targeting elements for postsynaptic density proteins ProSAP1/Shank2 and ProSAP2/Shank3. J. Neurochem. 92, 519-524 (2005).

45. Kim, E. \& Sheng, M. PDZ domain proteins of synapses. Nat. Rev. Neurosci. $\mathbf{5}$ 771-781 (2004).

46. Bonaglia, M. C. et al. Disruption of the ProSAP2 gene in a t(12:22)(q24.1; q13.3) is associated with the 22 q13.3 deletion syndrome. Am. J. Hum. Genet. 69, 261-268 (2001).

47. Phelan, K. \& McDermid, H. E. The 22q13.3 deletion syndrome (PhelanMcDermid Syndrome). Mol. Syndromol. 2, 186-201 (2012).

48. Watt, J. L. et al. A familial pericentric inversion of chromosome 22 with a recombinant subject illustrating a 'pure' partial monosomy syndrome. J. Med. Genet. 22, 283-287 (1985).

49. Leblond, C. S. et al. Meta-analysis of SHANK mutations in autism spectrum disorders: a gradient of severity in cognitive impairments. PLoS Genet. 10, e1004580 (2014).

50. Sato, D. et al. SHANK1 deletions in males with autism spectrum disorder. Am. J. Hum. Genet. 90, 879-887 (2012).

51. Wang, T. et al. De novo genic mutations among a Chinese autism spectrum disorder cohort. Nat. Commun. 7, 13316 (2016).

52. Lennertz, L. et al. A promoter variant of SHANK1 affects auditory working memory in schizophrenia patients and in subjects clinically at risk for psychosis. Eur. Arch. Psychiatry Clin. Neurosci. 262, 117-124 (2012).

53. Fromer, M. et al. De novo mutations in schizophrenia implicate synaptic networks. Nature 506, 179-184 (2014).

54. Chilian, B. et al. Dysfunction of SHANK2 and CHRNA7 in a patient with intellectual disability and language impairment supports genetic epistasis of the two loci. Clin. Genet. 84, 560-565 (2013).

55. Leblond, C. S. et al. Genetic and functional analyses of SHANK2 mutations suggest a multiple hit model of autism spectrum disorders. PLoS Genet. 8, e1002521 (2012).

56. Schluth-Bolard, C. et al. Breakpoint mapping by next generation sequencing reveals causative gene disruption in patients carrying apparently balanced chromosome rearrangements with intellectual deficiency and/or congenital malformations. J. Med. Genet. 50, 144-150 (2013).

57. Peykov, S. et al. Identification and functional characterization of rare SHANK2 variants in schizophrenia. Mol. Psychiatry 20, 1489-1498 (2015).

58. Gauthier, J. et al. De novo mutations in the gene encoding the synaptic scaffolding protein SHANK3 in patients ascertained for schizophrenia. Proc. Natl Acad. Sci. USA 107, 7863-7868 (2010).

59. Han, K. et al. SHANK3 overexpression causes manic-like behaviour with unique pharmacogenetic properties. Nature 503, 72-77 (2013).

60. Zhou, Y. et al. Mice with Shank3 mutations associated with ASD and schizophrenia display both shared and distinct defects. Neuron 89, 147-162 (2016).

61. Cestra, G., Toomre, D., Chang, S. \& De Camilli, P. The Abl/Arg substrate ArgBP2/nArgBP2 coordinates the function of multiple regulatory mechanisms converging on the actin cytoskeleton. Proc. Natl Acad. Sci. USA 102, 1731-1736 (2005).

62. Murase, $\mathrm{K}$. et al. Cell biological characterization of a multidomain adaptor protein, ArgBP2, in epithelial NMuMG cells, and identification of a novel short isoform. Med. Mol. Morphol. 45, 22-28 (2012).

63. Wang, B., Golemis, E. A. \& Kruh, G. D. ArgBP2, a multiple Src homology 3 domain-containing, Arg/Abl-interacting protein, is phosphorylated in V-Abltransformed cells and localized in stress fibers and cardiocyte Z-disks. J. Biol. Chem. 272, 17542-17550 (1997).

64. Yuan, Z. Q. et al. ArgBP2gamma interacts with Akt and p21-activated kinase-1 and promotes cell survival. J. Biol. Chem. 280, 21483-21490 (2005).

65. Zhang, Q. et al. Impaired dendritic development and memory in Sorbs2 knock-out mice. J. Neurosci. 36, 2247-2260 (2016).

66. Lein, E. S. et al. Genome-wide atlas of gene expression in the adult mouse brain. Nature 445, 168-176 (2007).

67. Strakowski, S. M., Delbello, M. P. \& Adler, C. M. The functional neuroanatomy of bipolar disorder: a review of neuroimaging findings. Mol. Psychiatry 10, 105-116 (2005).

68. Zucconi, A., Dente, L., Santonico, E., Castagnoli, L. \& Cesareni, G. Selection of ligands by panning of domain libraries displayed on phage lambda reveals new potential partners of synaptojanin 1. J. Mol. Biol. 307, 1329-1339 (2001).

69. Soubeyran, P., Barac, A., Szymkiewicz, I. \& Dikic, I. Cbl-ArgBP2 complex mediates ubiquitination and degradation of c-Abl. Biochem. J. 370, 29-34 (2003). 
70. Eden, S., Rohatgi, R., Podtelejnikov, A. V., Mann, M. \& Kirschner, M. W. Mechanism of regulation of WAVE1-induced actin nucleation by Rac1 and Nck. Nature 418, 790-793 (2002).

71. Innocenti, M. et al. Abi1 is essential for the formation and activation of a WAVE2 signalling complex. Nat. Cell Biol. 6, 319-327 (2004).

72. Proepper, C. et al. Abelson interacting protein 1 (Abi-1) is essential for dendrite morphogenesis and synapse formation. EMBO J. 26, 1397-1409 (2007)

73. Lee, S. E. et al. nArgBP2 regulates excitatory synapse formation by controlling dendritic spine morphology. Proc. Natl Acad. Sci. USA 113, 6749-6754 (2016).

74. Feng G. US patent $20,100,077,493$ (2010). Genes and pathways involved in bipolar disorder.

75. Maren, S., Phan, K. L. \& Liberzon, I. The contextual brain: implications for fear conditioning, extinction and psychopathology. Nat. Rev. Neurosci. 14 417-428 (2013).

76. Chen, C. Y. et al. Rescue of the genetically engineered Cul4b mutant mouse as a potential model for human X-linked mental retardation. Hum. Mol. Genet. 21, 4270-4285 (2012).

77. Powell, A. D. et al. Rapid reversal of impaired inhibitory and excitatory transmission but not spine dysgenesis in a mouse model of mental retardation. J. Physiol. 590, 763-776 (2012).

78. Shi, S. Obsessive compulsive symptoms in bipolar disorder patients: a comorbid disorder or a subtype of bipolar disorder? Shanghai Arch. Psychiatry 27, 249-251 (2015)

79. Borue, $X$. et al. Longitudinal course of bipolar disorder in youth with highfunctioning autism spectrum disorder. J. Am. Acad. Child. Adolesc. Psychiatry 55, 1064-1072.e6 (2016).

80. Khanzada, N. S., Butler, M. G. \& Manzardo, A. M. Gene analytics pathway analysis and genetic overlap among autism spectrum disorder, bipolar disorder and schizophrenia. Int J. Mol. Sci. 18, 527 (2017).

81. Nemeroff, C. B. Comorbidity of mood and anxiety disorders: the rule, not the exception? Am. J. Psychiatry 159, 3-4 (2002).

82. Cross-Disorder Group of the Psychiatric Genomics C, Lee, S. H. et al. Genetic relationship between five psychiatric disorders estimated from genomewide SNPs. Nat. Genet. 45, 984-994 (2013).

83. Woodbury-Smith, M. et al. Combined genome-wide linkage and targeted association analysis of head circumference in autism spectrum disorder families. J. Neurodev. Disord. 9, 5 (2017).

84. Zhao, H. \& Nyholt, D. R. Gene-based analyses reveal novel genetic overlap and allelic heterogeneity across five major psychiatric disorders. Hum. Genet. 136, 263-274 (2017).

85. Ryu, S. et al. Interaction between genetic variants of DLGAP3 and SLC1A1 affecting the risk of atypical antipsychotics-induced obsessivecompulsive symptoms. Am. J. Med. Genet. B Neuropsychiatr. Genet. 156B 949-959 (2011).

86. Durand, C. M. et al. Mutations in the gene encoding the synaptic scaffolding protein SHANK3 are associated with autism spectrum disorders. Nat. Genet. 39, 25-27 (2007).

87. de Sena Cortabitarte, A. et al. Investigation of SHANK3 in schizophrenia. Am. J. Med. Genet. B Neuropsychiatr. Genet. 174, 390-398 (2017).

88. Roselli, F., Livrea, P. \& Almeida, O. F. CDK5 is essential for soluble amyloid beta-induced degradation of GKAP and remodeling of the synaptic actin cytoskeleton. PLOS ONE 6, e23097 (2011).

89. Schutt, J., Falley, K., Richter, D., Kreienkamp, H. J. \& Kindler, S. Fragile X mental retardation protein regulates the levels of scaffold proteins and glutamate receptors in postsynaptic densities. J. Biol. Chem. 284, 25479-25487 (2009).

90. Liu, S., Zhang, Y., Bian, H. \& Li, X. Gene expression profiling predicts pathways and genes associated with Parkinson's disease. Neurol. Sci. 37, 73-79 (2016).

91. Li, J. M. et al. Exonic resequencing of the DLGAP3 gene as a candidate gene for schizophrenia. Psychiatry Res. 208, 84-87 (2013).

92. Minocherhomii, S. et al. Epigenetic remodelling and dysregulation of DLGAP4 is linked with early-onset cerebellar ataxia. Hum. Mol. Genet. 23 6163-6176 (2014).

93. Kim, Y. et al. Bipolar disorder associated microRNA, miR-1908-5p, regulates the expression of genes functioning in neuronal glutamatergic synapses. Exp. Neurobiol. 25, 296-306 (2016).
94. Roselli, F., Hutzler, P., Wegerich, Y., Livrea, P. \& Almeida, O. F. Disassembly of shank and homer synaptic clusters is driven by soluble beta-amyloid(1-40) through divergent NMDAR-dependent signalling pathways. PLOS ONE 4, e6011 (2009).

95. Pham, E. et al. Progressive accumulation of amyloid-beta oligomers in Alzheimer's disease and in amyloid precursor protein transgenic mice is accompanied by selective alterations in synaptic scaffold proteins. FEBS $\mathrm{J}$. 277, 3051-3067 (2010).

96. Berkel, S. et al. Mutations in the SHANK2 synaptic scaffolding gene in autism spectrum disorder and mental retardation. Nat. Genet. 42, 489-491 (2010).

97. Pappas, A. L. et al. Deficiency of Shank2 causes mania-like behavior that responds to mood stabilizers. JCl Insight 2, e92052 (2017).

98. Noor, A. et al. Copy number variant study of bipolar disorder in Canadian and UK populations implicates synaptic genes. Am. J. Med. Genet. B Neuropsychiatr. Genet. 165B, 303-313 (2014)

99. Gong, Y., Lippa, C. F., Zhu, J., Lin, Q. \& Rosso, A. L. Disruption of glutamate receptors at Shank-postsynaptic platform in Alzheimer's disease. Brain Res. 1292, 191-198 (2009).

100. Monteiro, P. \& Feng, G. SHANK proteins: roles at the synapse and in autism spectrum disorder. Nat. Rev. Neurosci. 18, 147-157 (2017).

101. Peca, J. et al. Shank3 mutant mice display autistic-like behaviours and striatal dysfunction. Nature 472, 437-442 (2011)

102. Connolly, S., Anney, R., Gallagher, L. \& Heron, E. A. A genome-wide investigation into parent-of-origin effects in autism spectrum disorder identifies previously associated genes including SHANK3. Eur. J. Hum. Genet. 25 234-239 (2017).

103. Gauthier, J. et al. Novel de novo SHANK3 mutation in autistic patients. Am. J. Med. Genet. B Neuropsychiatr. Genet. 150b, 421-424 (2009).

104. Moessner, R. et al. Contribution of SHANK3 mutations to autism spectrum disorder. Am. J. Hum. Genet. 81, 1289-1297 (2007).

105. Zhang, Y. et al. Expression of SHANK3 in the temporal neocortex of patients with intractable temporal epilepsy and epilepsy rat models. Cell. Mol. Neurobiol. 37, 857-867 (2017).

106. Holder, J. L. Jr \& Quach, M. M. The spectrum of epilepsy and electroencephalographic abnormalities due to SHANK3 loss-of-function mutations. Epilepsia 57, 1651-1659 (2016).

107. Jaramillo, T. C. et al. Altered striatal synaptic function and abnormal behaviour in Shank3 exon4-9 deletion mouse model of autism. Autism Res. 9, 350-375 (2016).

108. Lee, Y. et al. Integrative analysis of brain region-specific Shank3 interactomes for understanding the heterogeneity of neuronal pathophysiology related to SHANK3 mutations. Front. Mol. Neurosci. 10, 110 (2017).

109. Failla, P. et al. Schizophrenia in a patient with subtelomeric duplication of chromosome 22q. Clin. Genet. 71, 599-601 (2007).

110. Wang, X. et al. Synaptic dysfunction and abnormal behaviors in mice lacking major isoforms of Shank3. Hum. Mol. Genet. 20, 3093-3108 (2011).

111. Verhoeven, W. M., Egger, J. I., Willemsen, M. H., de Leijer, G. J. \& Kleefstra, T. Phelan-McDermid syndrome in two adult brothers: atypical bipolar disorder as its psychopathological phenotype? Neuropsychiatr. Dis. Treat. 8, 175-179 (2012).

112. Phelan, M. C. Deletion 22q13.3 syndrome. Orphanet J. Rare Dis. 3, 14 (2008).

113. Girard, S. L. et al. Mutation burden of rare variants in schizophrenia candidate genes. PLOS ONE 10, e0128988 (2015).

114. Grabrucker, S. et al. The PSD protein ProSAP2/Shank3 displays synaptonuclear shuttling which is deregulated in a schizophrenia-associated mutation. Exp. Neurol. 253, 126-137 (2014).

115. Sundararajan, T. Manzardo, A. M. \& Butler, M. G. Functional analysis of schizophrenia genes using GeneAnalytics program and integrated databases. Gene 641, 25-34 (2018).

116. Denayer, A. et al. Neuropsychopathology in 7 patients with the 22q13 deletion syndrome: presence of bipolar disorder and progressive loss of skills. Mol. Syndromol. 3, 14-20 (2012).

117. Vucurovic, K. et al. Bipolar affective disorder and early dementia onset in a male patient with SHANK3 deletion. Eur. J. Med. Genet. 55, 625-629 (2012).

118. Adams, L. J. et al. A susceptibility locus for bipolar affective disorder on chromosome 4q35. Am. J. Hum. Genet. 62, 1084-1091 (1998). 